\title{
Minimally doubled fermions and their renormalization
}

\section{Stefano Capitani*}

Institut für Kernphysik, Becher Weg 45, University of Mainz, D-55099 Mainz, Germany

E-mail: capitan@kph.uni-mainz.de

\section{Michael Creutz}

Physics Department, Brookhaven National Laboratory, Upton, NY 11973, USA

E-mail: mikedlatticeguy. net

\section{Johannes Weber ${ }^{\dagger}$}

Graduate School of Pure and Applied Physics, Tsukuba University, Tsukuba, Ibaraki, Japan

E-mail: weberjekph.uni-mainz.de

\section{Hartmut Wittig}

Institut für Kernphysik, Becher Weg 45, University of Mainz, D-55099 Mainz, Germany

E-mail: wittigakph.uni-mainz.de

\begin{abstract}
Minimally doubled fermions have been proposed as a strictly local discretization of the QCD quark action, which also preserves chiral symmetry at finite cut-off. We study the renormalization and mixing properties of two particular realizations of minimally doubled fermions in lattice perturbation theory at one loop, and we construct conserved axial currents which have a simple form involving only nearest-neighbours sites. We also introduce a notation which allows a unified description of the renormalization properties of both actions.
\end{abstract}

The XXVIII International Symposium on Lattice Field Theory, Lattice2010

June 14-19, 2010

Villasimius, Italy

\footnotetext{
${ }^{*}$ Speaker.

${ }^{\dagger}$ Presenter of the poster.
} 


\section{Introduction}

We report on our analytic studies of the renormalization properties of Boriçi-Creutz [1, 2, 3, 4] and Karsten-Wilczek [5, 6] fermions (see [7, 8, 9], and references therein), two particular realizations of minimally doubled fermions. ${ }^{1}$ These actions preserve an exact chiral symmetry for a degenerate doublet of quarks, and at the same time they remain strictly local, so that they are much cheaper to simulate than Ginsparg-Wilson fermions. They could then become a cost-effective realization of chiral symmetry at nonzero lattice spacing. This $U(1) \otimes U(1)$ chiral symmetry, which is of the same form as in the continuum, protects the quark mass from additive renormalization. As we have also verified at one loop, the renormalization of the quark mass has the same form as, say, overlap or staggered fermions.

It is noteworthy that using minimally doubled fermions one can construct a conserved axial current which has a simple expression, involving only nearest-neighbour sites (see Section 6). These actions are then among the very few lattice discretizations which provide a simple (ultralocal) expression for a conserved axial current.

It is natural to compare these realizations of minimally doubled fermions with staggered fermions, which preserve the same $U(1) \otimes U(1)$ chiral symmetry and are also ultralocal and comparably cheap. The advantage of Boriçi-Creutz and Karsten-Wilczek fermions is that they contain 2 flavours instead of 4, and thus they do not require any uncontrolled extrapolation to 2 physical light flavours [11, 12]. Moreover, the construction of fermionic operators is much easier than for staggered fermions, where there is also a complicated intertwining of spin and flavour. Minimally doubled actions look then ideal for $N_{f}=2$ simulations. ${ }^{2}$

\section{Actions}

The free Dirac operator of Boriçi-Creutz fermions is given in momentum space by

$$
D(p)=i \sum_{\mu}\left(\gamma_{\mu} \sin p_{\mu}+\gamma_{\mu}^{\prime} \cos p_{\mu}\right)-2 i \Gamma+m_{0}
$$

where

$$
\Gamma=\frac{1}{2}\left(\gamma_{1}+\gamma_{2}+\gamma_{3}+\gamma_{4}\right) \quad\left(\Gamma^{2}=1\right)
$$

and

$$
\gamma_{\mu}^{\prime}=\Gamma \gamma_{\mu} \Gamma=\Gamma-\gamma_{\mu}
$$

$D(p)$ vanishes at $p_{1}=(0,0,0,0)$ and $p_{2}=(\pi / 2, \pi / 2, \pi / 2, \pi / 2)$, and can also be seen as a linear combination of two physically equivalent naive fermions (one of them translated in momentum space).

The free Karsten-Wilczek Dirac operator is given in momentum space by

$$
D(p)=i \sum_{\mu=1}^{4} \gamma_{\mu} \sin p_{\mu}+i \gamma_{4} \sum_{k=1}^{3}\left(1-\cos p_{k}\right)
$$

\footnotetext{
${ }^{1}$ For recent developments, see also [10].

${ }^{2}$ They remain rather convenient also for $N_{f}=2+1$ and $N_{f}=2+1+1$ simulations. The second doublet of minimally doubled quarks will contain chirality-breaking terms in order to give different masses to the $s$ and $c$ quarks, however this is not so important for these larger masses.
} 
and its zeros are instead at $p_{1}=(0,0,0,0)$ and $p_{2}=(0,0,0, \pi)$.

The two zeros of these actions, corresponding to the physical flavours, select a special direction in euclidean spacetime, identified by the line that connects them. It is easy to see that in the BoriçiCreutz case the matrix $\Gamma$ selects as a special direction the major hypercube diagonal, while in the Karsten-Wilczek case is the temporal direction which becomes the special one.

As a consequence, hyper-cubic symmetry is broken, and these actions are symmetric only under the subgroup of the hyper-cubic group which preserves (up to a sign) the respective special direction. This opens the way to mixings of a new kind under renormalization. One of the main aims of our work is the investigation of the mixing patterns that appear in radiative corrections. We have elucidated the one-loop structure of these theories, and one of our main results is that everything is consistent at the one loop level, and the new mixings are very few.

We also remark that, although the distance between the two zeros is the same $\left(p_{2}^{2}-p_{1}^{2}=\pi^{2}\right)$, these two realizations of minimally doubled fermions are not equivalent.

\section{Counterterms}

Each of the two actions (2.1) and (2.4) does not contain all possible operators which are invariant under the subgroup of the hyper-cubic group preserving its respective special direction. Radiative corrections then generate new contributions whose form is not matched by any term in the original bare actions. It becomes necessary to introduce counterterms to the bare actions in order to obtain a consistent renormalized theory. Enforcing the consistency requirement will allow us to uniquely determine the coefficients of these counterterms. ${ }^{3}$

One must add to the bare actions all possible counterterms allowed by the remnant symmetries. Moreover, counterterms are needed also in the pure gauge part of the actions of minimally doubled fermions. The reason for this is that, although at the bare level the breaking of hyper-cubic symmetry happens only in the fermionic parts of the actions, in the renormalized theory it propagates (via the interactions between quarks and gluons) also to the pure gauge sector.

We consider the massless case $m_{0}=0$, and note that chiral symmetry strongly restricts the number of possible counterterms. It turns out that there is only one possible dimension-four fermionic counterterm, which for Boriçi-Creutz fermions is written in continuum form as $\bar{\psi} \Gamma \sum_{\mu} D_{\mu} \psi$. A possible discretization for it has a form similar to the hopping term in the action:

$$
c_{4}\left(g_{0}\right) \frac{1}{2 a} \sum_{\mu}\left(\bar{\psi}(x) \Gamma U_{\mu}(x) \psi(x+a \widehat{\mu})-\bar{\psi}(x+a \widehat{\mu}) \Gamma U_{\mu}^{\dagger}(x) \psi(x)\right) .
$$

There is also one counterterm of dimension three,

$$
\frac{i c_{3}\left(g_{0}\right)}{a} \bar{\psi}(x) \Gamma \psi(x)
$$

\footnotetext{
${ }^{3}$ It is interesting in this respect to observe that an action which contains doublers will in general select some special direction, and hence require counterterms. However, the staggered fermion formulation is very clever, because it rearranges the 16 spin-flavour components of the 4 doublers on the corners of the unit hypercube. Thanks to this, no special direction arises, and thus no extra counterterms are needed for the simulation of staggered fermions. In the case of naive fermions the 16 doublers are also uniformly distributed in the Brillouin zone, and hence there is no special direction in this case too.
} 
which is already present in the bare Boriçi-Creutz action, but with a fixed coefficient $-2 / a$. The appearance of this counterterm means that in the general renormalized action the coefficient of the dimension-three operator must be kept general.

For Karsten-Wilczek fermions we find a similar situation. The only gauge-invariant fermionic counterterm of dimension four is

$$
\bar{\psi} \gamma_{4} D_{4} \psi
$$

and a suitable discretization of it is

$$
d_{4}\left(g_{0}\right) \frac{1}{2 a}\left(\bar{\psi}(x) \gamma_{4} U_{4}(x) \psi(x+a \widehat{4})-\bar{\psi}(x+a \widehat{4}) \gamma_{4} U_{4}^{\dagger}(x) \psi(x)\right) .
$$

The counterterm of dimension three is for this action

$$
\frac{i d_{3}\left(g_{0}\right)}{a} \bar{\psi}(x) \gamma_{4} \psi(x)
$$

(already present in the bare Karsten-Wilczek action, with a fixed coefficient).

The rules for the counterterm corrections to fermion propagators, needed for our one-loop calculations, can be easily derived. For external lines, they are given in momentum space respectively by

$$
-i c_{4}\left(g_{0}\right) \Gamma \sum_{v} p_{v}, \quad-\frac{i c_{3}\left(g_{0}\right)}{a} \Gamma
$$

for Boriçi-Creutz fermions, and by

$$
-i d_{4}\left(g_{0}\right) \gamma_{4} p_{4}, \quad-\frac{i d_{3}\left(g_{0}\right)}{a} \gamma_{4}
$$

for Karsten-Wilczek fermions.

The gluonic counterterms must be of the form trFF, but with nonconventional choices of the indices, reflecting the breaking of the hyper-cubic symmetry. It turns out that there is only one purely gluonic counterterm, which for the Boriçi-Creutz action can be written in continuum form as

$$
c_{P}\left(g_{0}\right) \sum_{\lambda \rho \tau} \operatorname{trF}{ }_{\lambda \rho}(\mathrm{x}) \mathrm{F}_{\rho \tau}(\mathrm{x})
$$

At one loop this counterterm is relevant only for gluon propagators. Denoting the fixed external indices at their ends with $\mu$ and $v$, all possible lattice discretizations of this counterterm give in momentum space the same Feynman rule:

$$
-c_{P}\left(g_{0}\right)\left[\left(p_{\mu}+p_{v}\right) \sum_{\lambda} p_{\lambda}-p^{2}-\delta_{\mu v}\left(\sum_{\lambda} p_{\lambda}\right)^{2}\right] .
$$

Contributions of this kind must be taken into account for a correct renormalization of the vacuum polarization (see Section 5).

In the case of Karsten-Wilczek fermions the counterterm which needs to be introduced can be written in continuum form as

$$
d_{P}\left(g_{0}\right) \sum_{\rho \lambda} \operatorname{tr} \mathrm{F}_{\rho \lambda}(\mathrm{x}) \mathrm{F}_{\rho \lambda}(\mathrm{x}) \delta_{\rho 4}
$$


The Feynman rule for the insertion of this counterterm in external gluon propagators reads

$$
-d_{P}\left(g_{0}\right)\left[p_{\mu} p_{v}\left(\delta_{\mu 4}+\delta_{v 4}\right)-\delta_{\mu v}\left(p^{2} \delta_{\mu 4} \delta_{v 4}+p_{4}^{2}\right)\right] .
$$

In perturbation theory the coefficients of all counterterms are functions of the coupling which start at order $g_{0}^{2}$. We will determine (at one loop) the coefficients of all fermionic and gluonic counterterms by requiring that the renormalized self-energy and vacuum polarization, respectively, assume their standard form (see Sections 4 and 5).

Counterterm interaction vertices are generated as well. However, these vertex insertions are at least of order $g_{0}^{3}$, and thus they cannot contribute to the one-loop amplitudes that we study here. We also want to emphasize that counterterms not only provide additional Feynman rules for the calculation of loop amplitudes. They can also modify Ward identities and hence, in particular, contribute additional terms to the conserved currents (see Section 6).

\section{Determination of the fermionic counterterms}

Leaving aside for one moment the counterterms, the quark self-energy of a Boriçi-Creutz fermion is given at one loop by

$$
\Sigma\left(p, m_{0}\right)=i \not p \Sigma_{1}(p)+m_{0} \Sigma_{2}(p)+c_{1}\left(g_{0}\right) \cdot i \Gamma \sum_{\mu} p_{\mu}+c_{2}\left(g_{0}\right) \cdot i \frac{\Gamma}{a}
$$

where $^{4}$

$$
\begin{aligned}
& \Sigma_{1}(p)=\frac{g_{0}^{2}}{16 \pi^{2}} C_{F}\left[\log a^{2} p^{2}+6.80663+(1-\alpha)\left(-\log a^{2} p^{2}+4.792010\right)\right], \\
& \Sigma_{2}(p)=\frac{g_{0}^{2}}{16 \pi^{2}} C_{F}\left[4 \log a^{2} p^{2}-29.48729+(1-\alpha)\left(-\log a^{2} p^{2}+5.792010\right)\right], \\
& c_{1}\left(g_{0}\right)=1.52766 \cdot \frac{g_{0}^{2}}{16 \pi^{2}} C_{F}, \\
& c_{2}\left(g_{0}\right)=29.54170 \cdot \frac{g_{0}^{2}}{16 \pi^{2}} C_{F},
\end{aligned}
$$

with $C_{F}=\left(N_{c}^{2}-1\right) / 2 N_{c}$, and $\alpha$ denotes the gauge parameter in a general covariant gauge. The full inverse propagator at one loop can be written (without counterterms) as

$$
\Sigma^{-1}\left(p, m_{0}\right)=\left(1-\Sigma_{1}\right) \cdot\left\{i \not p+m_{0}\left(1-\Sigma_{2}+\Sigma_{1}\right)-i c_{1} \Gamma \sum_{\mu} p_{\mu}-\frac{i c_{2}}{a} \Gamma\right\} .
$$

We can only cast the renormalized propagator in the standard form

$$
\Sigma\left(p, m_{0}\right)=\frac{Z_{2}}{i \not p+Z_{m} m_{0}},
$$

where the wave-function and quark mass renormalization factors are given by

$$
Z_{2}=\left(1-\Sigma_{1}\right)^{-1}, \quad Z_{m}=1-\left(\Sigma_{2}-\Sigma_{1}\right)
$$

\footnotetext{
${ }^{4}$ For our calculations we have developed programs written in the algebraic computer language FORM [13, 14].
} 
provided that we employ the counterterms to cancel the Lorentz non-invariant factors $\left(c_{1}\right.$ and $\left.c_{2}\right)$.

The term proportional to $c_{1}$ can be eliminated by using the dimension-four counterterm, $\bar{\psi} \Gamma \sum_{\mu} D_{\mu} \psi$, while the term proportional to $c_{2}$ can be eliminated using the dimension-three counterterm, $1 / a \bar{\psi} \Gamma \psi$. This amounts to applying the insertions of eqs. (3.6) and (3.7). We thus determine in this way that at one loop, for Boriçi-Creutz fermions,

$$
c_{3}\left(g_{0}\right)=29.54170 \cdot \frac{g_{0}^{2}}{16 \pi^{2}} C_{F}+O\left(g_{0}^{4}\right), \quad c_{4}\left(g_{0}\right)=1.52766 \cdot \frac{g_{0}^{2}}{16 \pi^{2}} C_{F}+O\left(g_{0}^{4}\right) .
$$

Things work out very similarly for Karsten-Wilczek fermions. In this case the inverse propagator at one loop (without counterterms) is

$$
\Sigma^{-1}\left(p, m_{0}\right)=\left(1-\Sigma_{1}\right) \cdot\left(i p p+m_{0}\left(1-\Sigma_{2}+\Sigma_{1}\right)-i d_{1} \gamma_{4} p_{4}-\frac{i d_{2}}{a} \gamma_{4}\right)
$$

where

$$
\begin{aligned}
& \Sigma_{1}(p)=\frac{g_{0}^{2}}{16 \pi^{2}} C_{F}\left[\log a^{2} p^{2}+9.24089+(1-\alpha)\left(-\log a^{2} p^{2}+4.792010\right)\right], \\
& \Sigma_{2}(p)=\frac{g_{0}^{2}}{16 \pi^{2}} C_{F}\left[4 \log a^{2} p^{2}-24.36875+(1-\alpha)\left(-\log a^{2} p^{2}+5.792010\right)\right], \\
& d_{1}\left(g_{0}\right)=-0.12554 \cdot \frac{g_{0}^{2}}{16 \pi^{2}} C_{F} \\
& d_{2}\left(g_{0}\right)=-29.53230 \cdot \frac{g_{0}^{2}}{16 \pi^{2}} C_{F} .
\end{aligned}
$$

By using the appropriate counterterms $\bar{\psi} \gamma_{4} D_{4} \psi$ and $1 / a \bar{\psi} \gamma_{4} \psi$ the renormalized propagator can be written in the standard form. Then, at one loop we obtain

$$
d_{3}\left(g_{0}\right)=-29.53230 \cdot \frac{g_{0}^{2}}{16 \pi^{2}} C_{F}+O\left(g_{0}^{4}\right), \quad d_{4}\left(g_{0}\right)=-0.12554 \cdot \frac{g_{0}^{2}}{16 \pi^{2}} C_{F}+O\left(g_{0}^{4}\right) .
$$

One may expect that the above subtraction procedure can be carried out systematically at every order of perturbation theory. After the subtractions via the appropriate counterterms are properly taken into account, the extra terms appearing in the self-energy disappear.

\section{Determination of the gluonic counterterms}

Leaving aside for one moment the counterterms, the contribution of the fermionic loops to the one-loop vacuum polarization of Boriçi-Creutz fermions comes out from our calculations as

$$
\begin{aligned}
\Pi_{\mu \nu}^{(f)}(p)= & \left(p_{\mu} p_{v}-\delta_{\mu v} p^{2}\right)\left[\frac{g_{0}^{2}}{16 \pi^{2}} C_{2}\left(-\frac{8}{3} \log p^{2} a^{2}+23.6793\right)\right] \\
& -\left(\left(p_{\mu}+p_{v}\right) \sum_{\lambda} p_{\lambda}-p^{2}-\delta_{\mu v}\left(\sum_{\lambda} p_{\lambda}\right)^{2}\right) \frac{g_{0}^{2}}{16 \pi^{2}} C_{2} \cdot 0.9094
\end{aligned}
$$


where $\operatorname{Tr}\left(\mathrm{t}^{\mathrm{a}} \mathrm{t}^{\mathrm{b}}\right)=\mathrm{C}_{2} \delta^{\mathrm{ab}}$. For Karsten-Wilczek fermions the corresponding result is

$$
\begin{aligned}
\Pi_{\mu v}^{(f)}(p)= & \left(p_{\mu} p_{v}-\delta_{\mu v} p^{2}\right)\left[\frac{g_{0}^{2}}{16 \pi^{2}} C_{2}\left(-\frac{8}{3} \log p^{2} a^{2}+19.99468\right)\right] \\
& -\left(p_{\mu} p_{v}\left(\delta_{\mu 4}+\delta_{v 4}\right)-\delta_{\mu v}\left(p^{2} \delta_{\mu 4} \delta_{v 4}+p_{4}^{2}\right)\right) \frac{g_{0}^{2}}{16 \pi^{2}} C_{2} \cdot 12.69766
\end{aligned}
$$

We notice the appearance of non-standard terms, compared with e.g. Wilson fermions. These new terms break hyper-cubic symmetry. It is remarkable that they still satisfy the Ward identity $p^{\mu} \Pi_{\mu v}^{(f)}(p)=0$.

At this stage we can employ the gluonic counterterms, which correspond to the insertions in the gluon propagator according to eqs. (3.9) and (3.11), to cancel the hyper-cubic-breaking terms in the vacuum polarization. The coefficients of these counterterms are hence determined as

$$
c_{P}\left(g_{0}\right)=-0.9094 \cdot \frac{g_{0}^{2}}{16 \pi^{2}} C_{2}+O\left(g_{0}^{4}\right), \quad d_{P}\left(g_{0}\right)=-12.69766 \cdot \frac{g_{0}^{2}}{16 \pi^{2}} C_{2}+O\left(g_{0}^{4}\right) .
$$

It is also very important to remark that no power-divergences $\left(1 / a^{2}\right.$ or $\left.1 / a\right)$ show up in our results for the vacuum polarization.

\section{Conserved currents}

We have also calculated the renormalization of the local Dirac bilinears. We have found that no mixings occur for the scalar and pseudoscalar densities and the tensor current. For the vector and axial currents instead a mixing can be seen, which is a consequence of the breaking of hypercubic invariance, and their renormalization factors $Z_{V}$ and $Z_{A}$ are thus are not equal to one (for their numerical values see Section 8). These local currents are indeed not conserved. Using chiral Ward identities we have then derived the expressions of the conserved currents, which are protected from renormalization.

As we have previously remarked, the counterterms influence the expressions of the conserved currents. It is easy to see that the counterterm of dimension three does not modify the Ward identities, and is irrelevant in this regard. On the contrary, the dimension-four counterterm

$$
\frac{c_{4}\left(g_{0}\right)}{4} \sum_{\mu} \sum_{v}\left(\bar{\psi}(x) \gamma_{v} U_{\mu}(x) \psi(x+a \widehat{\mu})+\bar{\psi}(x+a \widehat{\mu}) \gamma_{v} U_{\mu}^{\dagger}(x) \psi(x)\right)
$$

generates new terms in the Ward identities and hence contributes to the conserved currents. The conserved axial current for Boriçi-Creutz fermions in the renormalized theory turns out to have the expression

$$
\begin{aligned}
A_{\mu}^{\mathrm{c}}(x)= & \frac{1}{2}\left(\bar{\psi}(x)\left(\gamma_{\mu}+i \gamma_{\mu}^{\prime}\right) \gamma_{5} U_{\mu}(x) \psi(x+a \widehat{\mu})+\bar{\psi}(x+a \widehat{\mu})\left(\gamma_{\mu}-i \gamma_{\mu}^{\prime}\right) \gamma_{5} U_{\mu}^{\dagger}(x) \psi(x)\right) \\
& +\frac{c_{4}\left(g_{0}\right)}{2}\left(\bar{\psi}(x) \Gamma \gamma_{5} U_{\mu}(x) \psi(x+a \widehat{\mu})+\bar{\psi}(x+a \widehat{\mu}) \Gamma \gamma_{5} U_{\mu}^{\dagger}(x) \psi(x)\right) .
\end{aligned}
$$


For Karsten-Wilczek fermions, application of the chiral Ward identities gives for the conserved axial current

$$
\begin{aligned}
A_{\mu}^{\mathrm{c}}(x)= & \frac{1}{2}\left(\bar{\psi}(x)\left(\gamma_{\mu}-i \gamma_{4}\left(1-\delta_{\mu 4}\right)\right) \gamma_{5} U_{\mu}(x) \psi(x+a \widehat{\mu})\right. \\
& \left.+\bar{\psi}(x+a \widehat{\mu})\left(\gamma_{\mu}+i \gamma_{4}\left(1-\delta_{\mu 4}\right)\right) \gamma_{5} U_{\mu}^{\dagger}(x) \psi(x)\right) \\
& +\frac{d_{4}\left(g_{0}\right)}{2}\left(\bar{\psi}(x) \gamma_{4} \gamma_{5} U_{4}(x) \psi(x+a \widehat{4})+\bar{\psi}(x+a \widehat{4}) \gamma_{4} \gamma_{5} U_{4}^{\dagger}(x) \psi(x)\right) .
\end{aligned}
$$

The conserved vector currents can be obtained by simply dropping the $\gamma_{5}$ matrices from the above expressions. We remark that the vector current is isospin-singlet, representing the conservation of fermion number (as also discussed in [15]). The axial current, however, is a non-singlet because the doubled fermions have opposite chirality. All these currents have a very simple structure, which involves only nearest-neighbour sites.

We have computed the renormalization of these point-split currents, and verified that is one. As all four cases are very similar, we briefly discuss here the conserved vector current for BoriçiCreutz fermions, for which the sum of the "standard" diagrams (vertex, sails and operator tadpole, without the counterterm) gives

$$
\frac{g_{0}^{2}}{16 \pi^{2}} C_{F} \gamma_{\mu}\left[-\log a^{2} p^{2}-6.80664+(1-\alpha)\left(\log a^{2} p^{2}-4.79202\right)\right]+c_{1}^{c v}\left(g_{0}\right) \Gamma
$$

The value of the coefficient of the mixing is $c_{1}^{c v}\left(g_{0}\right)=-1.52766 \cdot \frac{g_{0}^{2}}{16 \pi^{2}} C_{F}+O\left(g_{0}^{4}\right)$.

When one adds to this result the wave-function renormalization (that is, $\Sigma_{1}(p)$ of the quark self-energy), the term proportional to $\gamma_{\mu}$ is exactly cancelled. The mixing term, proportional to $\Gamma$, instead remains, because we have not yet taken into account the counterterm.

The part of the conserved vector current due to the counterterm corresponds to the last line of eq. (6.2). Its 1-loop contribution is quite easy to compute (since $c_{4}$ is already of order $g_{0}^{2}$ ), and is given by $c_{4}\left(g_{0}\right) \Gamma$. We now note that the value of $c_{4}$ is already known from the self-energy, and numerical inspection shows that $c_{4}\left(g_{0}\right)=-c_{1}^{c v}\left(g_{0}\right)$ (within the precision of our integration routines). Thus, the $\Gamma$ mixing term is finally cancelled. We emphasize that only this particular value of $c_{4}$, determined from the self-energy, does exactly this job.

We have thus obtained that the renormalization constant of these point-split currents is one, which confirms that they are conserved currents. Everything turns out to be consistent at the one loop level.

\section{Numerical simulations}

If we use the nearest-neighbour forward covariant derivative $\nabla_{\mu} \psi(x)=\frac{1}{a}\left[U_{\mu}(x) \psi(x+a \widehat{\mu})-\right.$ $\psi(x)]$ and the corresponding backward one $\nabla_{\mu}^{*}$, we can express the (bare) actions in position space in a rather compact form. It then becomes apparent that these two realizations of minimally doubled fermions bear a close formal resemblance to Wilson fermions:

$$
D_{\text {Wilson }}^{f}=\frac{1}{2}\left\{\sum_{\mu=1}^{4} \gamma_{\mu}\left(\nabla_{\mu}+\nabla_{\mu}^{*}\right)-\operatorname{ar} \sum_{\mu=1}^{4} \nabla_{\mu}^{*} \nabla_{\mu}\right\}
$$




$$
\begin{aligned}
D_{\mathrm{BC}}^{f} & =\frac{1}{2}\left\{\sum_{\mu=1}^{4} \gamma_{\mu}\left(\nabla_{\mu}+\nabla_{\mu}^{*}\right)+i a \sum_{\mu=1}^{4} \gamma_{\mu}^{\prime} \nabla_{\mu}^{*} \nabla_{\mu}\right\}, \\
D_{\mathrm{KW}}^{f} & =\frac{1}{2}\left\{\sum_{\mu=1}^{4} \gamma_{\mu}\left(\nabla_{\mu}+\nabla_{\mu}^{*}\right)-i a \gamma_{4} \sum_{k=1}^{3} \nabla_{k}^{*} \nabla_{k}\right\} .
\end{aligned}
$$

All these three formulations contain a dimension-five operator in the bare action, and so we expect leading lattice artefacts to be of order $a$. However, for minimally doubled fermions these effects could numerically be small, if the results of [16] are to be believed.

We will not discuss here how to achieve one-loop (or nonperturbative) order $a$ improvement for these theories. The classification of all relevant independent operators could turn out to require a lengthy analysis. Notice that additional dimension-5 operators will occur not only in the quark sector (e.g., $\bar{\psi} \Gamma \sum_{\mu \nu} D_{\mu} D_{\nu} \psi$ ), but also in the pure gauge part (e.g., $\sum_{\mu \nu \lambda} F_{\mu \nu} D_{\lambda} F_{\mu \nu}$ ). Indeed, when Lorentz invariance is broken, the statement that only operators with even dimension can appear in the pure gauge action is no longer true.

We would now like to see what can be learned, from the one-loop calculations that we have carried out, regarding the numerical simulations of minimally doubled fermions. These simulations will have to employ the complete renormalized actions, including the counterterms.

The renormalized action for Boriçi-Creutz fermions in position space contains three counterterms and reads

$$
\begin{aligned}
S_{B C}^{f}=a^{4} \sum_{x} & \left\{\frac { 1 } { 2 a } \sum _ { \mu = 1 } ^ { 4 } \left[\bar{\psi}(x)\left(\gamma_{\mu}+c_{4}(\beta) \Gamma+i \gamma_{\mu}^{\prime}\right) U_{\mu}(x) \psi(x+a \widehat{\mu})\right.\right. \\
& \left.-\bar{\psi}(x+a \widehat{\mu})\left(\gamma_{\mu}+c_{4}(\beta) \Gamma-i \gamma_{\mu}^{\prime}\right) U_{\mu}^{\dagger}(x) \psi(x)\right] \\
& +\bar{\psi}(x)\left(m_{0}+\widetilde{c}_{3}(\beta) \frac{i \Gamma}{a}\right) \psi(x) \\
& \left.+\beta \sum_{\mu<v}\left(1-\frac{1}{N_{c}} \operatorname{Retr} \mathrm{P}_{\mu v}\right)+\mathrm{c}_{\mathrm{P}}(\beta) \sum_{\mu \nu \rho} \operatorname{tr}_{\mu \rho}^{\mathrm{lat}}(\mathrm{x}) \mathrm{F}_{\rho v}^{\mathrm{lat}}(\mathrm{x})\right\}
\end{aligned}
$$

where $F^{\text {lat }}$ is some lattice discretization of the field-strength tensor. We have here redefined the coefficient of the dimension-3 counterterm, using $\widetilde{c}_{3}(\beta)=-2+c_{3}(\beta)$ (which does not vanish at tree level). ${ }^{5}$

The renormalized action for Karsten-Wilczek fermions also contains three counterterms and reads

$$
\begin{aligned}
& S_{K W}^{f}=a^{4} \sum_{x}\left\{\frac { 1 } { 2 a } \sum _ { \mu = 1 } ^ { 4 } \left[\bar{\psi}(x)\left(\gamma_{\mu}\left(1+d_{4}(\beta) \delta_{\mu 4}\right)-i \gamma_{4}\left(1-\delta_{\mu 4}\right)\right) U_{\mu}(x) \psi(x+a \widehat{\mu})\right.\right. \\
&\left.-\bar{\psi}(x+a \widehat{\mu})\left(\gamma_{\mu}\left(1+d_{4}(\beta) \delta_{\mu 4}\right)+i \gamma_{4}\left(1-\delta_{\mu 4}\right)\right) U_{\mu}^{\dagger}(x) \psi(x)\right]
\end{aligned}
$$

\footnotetext{
${ }^{5}$ We assume that simulations will be carried out at very small values of $m_{0}$, so that our analysis of the counterterms, which assumes chiral symmetry, is essentially still valid. But note also that in our results of eqs. 4.1 and 4.10, obtained for general $m_{0}$, no new dimension-four terms proportional to this mass appear (apart from the standard one, $\Sigma_{2}$ ). Thus, at one loop we do not need further counterterms in additions to the three which we have found. This strongly suggests that our analysis of the counterterms remains valid even when chiral symmetry is broken.
} 


$$
\begin{aligned}
& +\bar{\psi}(x)\left(m_{0}+\widetilde{d}_{3}(\beta) \frac{i \gamma_{4}}{a}\right) \psi(x) \\
& \left.+\beta \sum_{\mu<v}\left(1-\frac{1}{N_{c}} \operatorname{Re} \operatorname{tr} \mathrm{P}_{\mu v}\right)\left(1+\mathrm{d}_{\mathrm{P}}(\beta) \delta_{\mu 4}\right)\right\}
\end{aligned}
$$

$\left(\widetilde{d_{3}}(\beta)=3+d_{3}(\beta)\right.$ has a non-zero value at tree level).

In perturbation theory the coefficients of the counterterms have the expansions

$$
\begin{array}{rrrr}
\widetilde{c}_{3}\left(g_{0}\right)=-2+c_{3}^{(1)} g_{0}^{2}+c_{3}^{(2)} g_{0}^{4}+\ldots ; & \widetilde{d}_{3}\left(g_{0}\right)=3+d_{3}^{(1)} g_{0}^{2}+d_{3}^{(2)} g_{0}^{4}+\ldots \\
c_{4}\left(g_{0}\right)= & c_{4}^{(1)} g_{0}^{2}+c_{4}^{(2)} g_{0}^{4}+\ldots ; & d_{4}\left(g_{0}\right)= & d_{4}^{(1)} g_{0}^{2}+d_{4}^{(2)} g_{0}^{4}+\ldots \\
c_{P}\left(g_{0}\right)= & c_{P}^{(1)} g_{0}^{2}+c_{P}^{(2)} g_{0}^{4}+\ldots ; & d_{P}\left(g_{0}\right)= & d_{P}^{(1)} g_{0}^{2}+d_{P}^{(2)} g_{0}^{4}+\ldots
\end{array}
$$

The same counterterms also appear at the nonperturbative level, and need to be taken into account for a consistent simulation of these fermions. Their nonperturbative determination is one the most important task for the near future. This can be achieved using suitable renormalization conditions, and it remains to be seen which ones will turn out to be more convenient in practice.

We have previously seen that in perturbation theory the four-dimensional fermionic counterterm is necessary for the proper construction of the conserved currents. Its coefficient, as determined from the one-loop self-energy, has exactly the right value for which the conserved currents remain unrenormalized. This suggests that one possible nonperturbative determination of $c_{4}$ (and $d_{4}$ ) can be accomplished by simulating matrix elements of the (unrenormalized) conserved current, and imposing (by tuning the coefficient) that the electric charge is one.

Another effect of radiative corrections is to move the poles of the quark propagator away from their tree-level positions. It is the task of the dimension-three counterterm, for the appropriate value of the coefficient $c_{3}$ (or $d_{3}$ ), to bring the two poles back to their original locations. These shifts of the poles can introduce oscillations in some hadronic correlation functions as a function of time separation (similarly to staggered fermions). Then one possible way to determine $c_{3}\left(d_{3}\right)$ is to tune it in appropriately chosen correlation functions until these oscillations are removed.

Such oscillations, familiar from the staggered formulation, come about since the underlying fermion field can create several different species, and these species occur in different regions of the Brillouin zone. It would be interesting to explore whether or not these oscillations could be cancelled by constructing hadronic operators spread over nearby neighbours [17].

It is important to remember that because the two species are of opposite chirality, the naive $\gamma_{5}$ matrix is physically a flavour non-singlet. The naive on-site pseudoscalar field $\bar{\psi} \gamma_{5} \psi$ can create only flavour non-singlet pseudoscalar states. To create the flavour-singlet pseudoscalar meson, which gets its mass from the anomaly, one needs to combine fields on nearby sites with appropriate phases.

We would like to stress that the breaking of hyper-cubic symmetry does not generate any sign problem for the Monte Carlo generation of configurations. The gauge action is real, and the eigenvalues of the Dirac operator come in complex conjugate pairs, so that the fermion determinant is always non-negative.

The purely gluonic counterterm for Boriçi-Creutz fermions introduces in the renormalized action operators of the kind $E \cdot B, E_{1} E_{2}, B_{2} B_{3}$ (and similar). In a hyper-cubic invariant theory, 
instead, only the standard terms $E^{2}$ and $B^{2}$ are allowed. Fixing the coefficient $c_{P}$ could then be done by measuring $\langle E \cdot B\rangle,\left\langle E_{1} E_{2}\right\rangle, \cdots$, and tuning $c_{P}$ in such a way that one (or more) of these expectation values is restored to its proper value pertinent to a hyper-cubic invariant theory, i.e. zero. These effects could turn out to be rather small, given that only the fermionic part of the treelevel action breaks hyper-cubic symmetry. It could also be that other derived quantities are more sensitive to this coefficient, and more suitable for its nonperturbative determination. In general one can look for Ward identities in which violations of the standard Lorentz invariant form, as functions of $c_{P}$, occur.

For Karsten-Wilczek fermions the purely gluonic counterterm introduces an asymmetry between Wilson loops containing temporal links relative to those involving spatial links only. One could then fix $d_{P}$ by computing a Wilson loop lying entirely in two spatial directions, and then equating its result to an ordinary Wilson loop which also has links in the time direction.

In the end only Monte Carlo simulations will reveal the actual amount of symmetry breaking. This could turn out to be large or small depending on the observable considered. One important such quantity is the mass splitting of the charged pions relative to the neutral pion. Indeed, since there is only a $U(1) \otimes U(1)$ chiral symmetry, the $\pi^{0}$ is massless, as the unique Goldstone boson (for $m_{0} \rightarrow 0$ ), but $\pi^{+}$and $\pi^{-}$are massive.

Furthermore, the magnitude of these symmetry-breaking effects could turn out to be substantially different for Boriçi-Creutz compared to Karsten-Wilczek fermions. Thus, one of these two actions could in this way be raised to become the preferred one for numerical simulations.

\section{A unifying notation for the two fermion discretizations}

By introducing a particular notation, some similarities between the two realizations of minimally doubled fermions can be revealed. This applies to the form of the action, operators and counterterms. For this purpose one can introduce a 4-component object $\Lambda_{\mu}$, defined as

$$
\Lambda_{\mu} \equiv\left\{\begin{array}{ll}
\delta_{\mu 4} & \text { Karsten-Wilczek } \\
\frac{1}{2} & \text { Boriçi-Creutz }
\end{array}, \quad(\Lambda \cdot \gamma) \equiv \begin{cases}\gamma^{4} & \text { Karsten-Wilczek } \\
\Gamma & \text { Boriçi-Creutz }\end{cases}\right.
$$

In both cases this object points from the zero of the action at the center of the Brillouin zone to the other zero (describing the second fermion, of opposite chirality).

At first we show that by means of this object one can cast both actions into similar (although non-equivalent) forms. Their free Dirac operators, as we have already seen in Section 7, contain the same naive fermion piece but a different dimension-five operator. The latter can be rewritten in this new notation as

$$
\begin{aligned}
D_{K W}^{(5)}(k) & \equiv \frac{2 i}{a} \sum_{\mu, v} \Lambda^{v} \gamma^{v} \sin ^{2} \frac{a p_{\mu}}{2}\left(1-\delta_{\mu v}\right) \\
D_{B C}^{(5)}(k) & \equiv-\frac{2 i}{a} \sum_{\mu, v} \Lambda^{v} \gamma^{v} \sin ^{2} \frac{a p_{\mu}}{2}\left(1-2 \delta_{\mu v}\right) .
\end{aligned}
$$

The factors $\left(1-\delta_{\mu v}\right)$ and $\left(1-2 \delta_{\mu v}\right)$ cannot be transformed into each other, and this illustrates that the two actions are inequivalent and must be distinguished (as we remarked in Section 2). 
Although the quark propagator cannot be cast into a uniform expression using this notation, this turns out to be possible for operators (e.g. local currents and counterterms), as well as some other results such as the expression for vacuum polarization. For example, the various counterterms that we have previously discussed can be easily cast in a completely unified way for the two actions. If we rewrite the three counterterms making use of the object $\Lambda_{\mu}$, the counterterms of dimension three appear as

$$
\bar{\imath}(x)(\Lambda \cdot \gamma) \psi(x)
$$

the fermionic ones of dimension four become

$$
\bar{\psi}(x)(\Lambda \cdot \gamma)(\Lambda \cdot D) \psi(x)
$$

and the gluonic ones are

$$
\sum_{\mu, v, \rho} \Lambda_{\mu} F_{\mu \rho} F_{\rho v} \Lambda_{v}
$$

Here (and in the following) objects written in this unified notation may differ by simple numerical coefficients from the corresponding quantities which we have previously used in the conventional notation.

Let us now consider the results of the one-loop calculation that we have presented in the previous Sections. One can rewrite the full self-energy (without counterterms) for both actions as

$$
\Sigma=i \not p \Sigma_{1}+m_{0} \Sigma_{2}+i \tilde{c}_{1}(\Lambda \cdot \gamma)(\Lambda \cdot p)+\tilde{c}_{2} \frac{i}{a}(\Lambda \cdot \gamma)
$$

with $\tilde{c}_{i}$ being given by either $c_{i}$ or $d_{i}$. Also the fermionic bilinears can be expressed in a unified form. Using the abbreviations $b=\frac{g_{0}^{2} C_{F}}{16 \pi^{2}}$ and $L=\log a^{2} p^{2}$, the results for the one-loop vertex diagram for the local scalar, vector and tensor bilinears are

$$
\begin{gathered}
C^{S}=b\left\{\begin{array}{l}
(-4 L+24.36875+(1-\alpha)(L-5.792010)) \\
(-4 L+29.48729+(1-\alpha)(L-5.792010))
\end{array} \begin{array}{l}
\text { Karsten-Wilczek } \\
\text { Boriçi-Creutz }
\end{array}\right. \\
C_{\mu}^{V}=b\left\{\begin{array}{l}
\gamma_{\mu}(-L+10.44610+(1-\alpha)(L-4.792010))-2.88914 \cdot \Lambda_{\mu}(\Lambda \cdot \gamma) \text { Karsten-Wilczek } \\
\gamma_{\mu}(-L+9.54612+(1-\alpha)(L-4.792010))-0.20074 \cdot \Lambda_{\mu}(\Lambda \cdot \gamma) \text { Boriçi-Creutz }
\end{array}\right. \\
C_{\mu \nu}^{T}=b\left\{\begin{array}{l}
\sigma_{\mu \nu}(4.17551+(1-\alpha)(L-3.792010)) \\
\sigma_{\mu \nu}(2.16548+(1-\alpha)(L-3.792010))
\end{array}\right) \begin{array}{l}
\text { Karsten-Wilczek } \\
\text { Boriçi-Creutz }
\end{array}
\end{gathered}
$$

For the conserved vector current, the sum of the standard proper diagrams (vertex, sails and operator tadpole) reads for the two actions

$$
b\left\{\begin{array}{l}
\gamma_{\mu}\left(-L-9.24089+(1-\alpha)(L-4.792010)+0.12554 \cdot \Lambda_{\mu}(\Lambda \cdot \gamma)\right. \text { Karsten-Wilczek } \\
\gamma_{\mu}(-L-6.80663+(1-\alpha)(L-4.792010))-3.05532 \cdot \Lambda_{\mu}(\Lambda \cdot \gamma) \text { Boriçi-Creutz }
\end{array} .\right.
$$

Perhaps one of the most striking examples of the convenience of this notation can be observed in the case of the vacuum polarization. The contribution of fermion loops to this quantity contains structures which break hyper-cubic symmetry. It can be written as

$$
\Pi_{\mu \nu}^{(f)}(p)=\Sigma_{3}\left(p_{\mu} p_{v}-p^{2} \delta_{\mu v}\right)+d_{g}\left((\Lambda \cdot p)\left(\Lambda_{\mu} p_{v}+\Lambda_{v} p_{\mu}\right)-\left(\Lambda_{\mu} \Lambda_{v} p^{2}+\delta_{\mu v}(\Lambda \cdot p)^{2}\right)\right)
$$


with the numerical results (as we have seen in Section 5)

$$
\begin{aligned}
& \Sigma_{3}\left(g_{0}^{2}\right)=\tilde{b}\left\{\begin{array}{l}
-\frac{8}{3} L+19.99468 \text { Karsten-Wilczek } \\
-\frac{8}{3} L+23.6793 \text { Boriçi-Creutz }
\end{array},\right. \\
& d_{g}\left(g_{0}^{2}\right)=\tilde{b}\left\{\begin{array}{l}
-12.69766 \text { Karsten-Wilczek } \\
-3.6376 \text { Boriçi-Creutz }
\end{array}\right.
\end{aligned}
$$

with $\tilde{b}=\frac{g_{0}^{2} C_{2}}{16 \pi^{2}}$ (Wilson fermions have $\Sigma_{3}=\tilde{b}\left(-\frac{4}{3} L+4.337002\right)$ and $\left.d_{g}=0\right)$. Thus, a single formula can describe the structures which arise in the calculation of the vacuum polarization for both actions.

With this notation we have thus shown that operator structures and results for Boriçi-Creutz and Karsten-Wilczek fermions, although distinct, share many common traits. As can be inspected in the above expressions, another remarkable feature appears to be that, after $\Lambda_{\mu}$ is introduced, the summed indices occur in pairs (like in the continuum), and also the free indices match exactly on both sides of equations. We do not know if this will always happen, also if one computes more complicated quantities.

Even without using the $\Lambda$ notation, we also discovered that the hyper-cubic-breaking terms of the vacuum polarization in eq. (8.12) can be put for both actions in the same algebraic form, namely

$$
p^{2}\left\{\gamma_{\mu}, \Gamma\right\}\left\{\gamma_{v}, \Gamma\right\}+\delta_{\mu v}\{\not p, \Gamma\}\{\not p, \Gamma\}-\frac{1}{2}\{\not p, \Gamma\}\left(\left\{\gamma_{\mu}, \not p\right\}\left\{\gamma_{v}, \Gamma\right\}+\left\{\gamma_{v}, \not p\right\}\left\{\gamma_{\mu}, \Gamma\right\}\right),
$$

where in the case of Karsten-Wilczek fermions $\Gamma$ must be replaced by $\gamma_{4} / 2$. This substitution is suggested by comparison of the standard relation $\Gamma=\frac{1}{4} \sum_{\mu}\left(\gamma_{\mu}+\gamma_{\mu}^{\prime}\right)$ of Boriçi-Creutz fermions with the formula $\gamma_{4}=\frac{1}{2} \sum_{\mu}\left(\gamma_{\mu}+\gamma_{\mu}^{\prime}\right)$ for Karsten-Wilczek fermions, expressing the symmetries of the action (as can be seen from the expression of the propagator, when one expands it around the second zero). Whether there is any deeper significance to this structural "equivalence" of the hyper-cubic-breaking structures in the vacuum polarizations remains an open question.

\section{Conclusions}

Boriçi-Creutz and Karsten-Wilczek fermions are described by a fully consistent renormalized quantum field theory. Three counterterms need to be added to the bare actions, and all their coefficients can be calculated either in perturbation theory (as we have shown), or nonperturbatively from Monte Carlo simulations (a task for the future, for which we have suggested some strategies). After these subtractions are consistently taken into account, the power divergence in the self-energy is eliminated, and no other power divergences occur for all quantities that we calculated.

We have argued that under reasonable assumptions and following the nonperturbative determination of these counterterms, no special features of these two realizations of minimally doubled fermions should hinder their successful Monte Carlo simulation.

Conserved vector and axial currents can be derived, and they have simple expressions which involve only nearest-neighbours sites. We have then here one of the very few cases where one can define a simple conserved axial current (also ultralocal). 
Finally, we would like to observe that this work is also an example of the usefulness of perturbation theory in helping to unfold theoretical aspects of (new) lattice formulations.

\section{Acknowledgments}

This work was supported by Deutsche Forschungsgemeinschaft (SFB443), the GSI HelmholtzZentrum für Schwerionenforschung, and the Research Centre "Elementary Forces and Mathematical Foundations" (EMG) funded by the State of Rhineland-Palatinate. MC was supported by contract number DE-AC02-98CH10886 with the U.S. Department of Energy. Accordingly, the U.S. Government retains a non-exclusive, royalty-free license to publish or reproduce the published form of this contribution, or allow others to do so, for U.S. Government purposes. MC is particularly grateful to the Alexander von Humboldt Foundation for support for multiple visits to the University of Mainz.

\section{References}

[1] M. Creutz, JHEP 04 (2008) 017, arXiv:0712.1201.

[2] A. Boriçi, Phys. Rev. D78 (2008) 074504, arXiv:0712.4401.

[3] M. Creutz, PoS LATTICE2008 (2008) 080, arXiv:0808.0014.

[4] A. Boriçi, PoS LATTICE2008 (2008) 231, arXiv:0812.0092.

[5] L.H. Karsten, Phys. Lett. B104 (1981) 315.

[6] F. Wilczek, Phys. Rev. Lett. 59 (1987) 2397.

[7] S. Capitani, J. Weber and H. Wittig, Phys. Lett. B681 (2009) 105, arXiv:0907.2825.

[8] S. Capitani, J. Weber and H. Wittig, PoS LATTICE2008 (2009) 075, arXiv:0910.2597.

[9] S. Capitani, M. Creutz, J. Weber and H. Wittig, JHEP 1009 (2010) 027, arXiv:1006.2009.

[10] M. Creutz and T. Misumi, (2010), arXiv:1007.3328.

[11] M. Creutz, Annals Phys. 324 (2009) 1573, arXiv:0901.0150.

[12] M. Creutz, PoS QCD-TNT09 (2009) 008, arXiv:0909.5101.

[13] J.A.M. Vermaseren, (2000), math-ph/0010025.

[14] J.A.M. Vermaseren, Nucl. Phys. Proc. Suppl. 183 (2008) 19, arXiv:0806.4080.

[15] B.C. Tiburzi, Phys. Rev.D82 (2010) 034511, arXiv:1006.0172.

[16] K. Cichy, J. Gonzalez Lopez, K. Jansen, A. Kujawa and A. Shindler, Nucl. Phys. B800 (2008) 94, arXiv:0802.3637.

[17] M. Creutz, (2010), arXiv:1009.3154. 